\title{
Nonextensive thermodynamic functions in the Schrödinger-Gibbs ensemble
}

\author{
J. L. Alonso, ${ }^{1,2,3}$ A. Castro, ${ }^{4}$ J. Clemente-Gallardo, ${ }^{1,2,3}$ J. C. Cuchí, ${ }^{5}$ P. Echenique, ${ }^{1,2,3,6}$ J. G. Esteve, ${ }^{1,2}$ and F. Falceto ${ }^{1,2}$ \\ ${ }^{1}$ Departamento de Física Teórica, Universidad de Zaragoza, Pedro Cerbuna 12, 50009 Zaragoza, Spain \\ ${ }^{2}$ Instituto de Biocomputación y Física de Sistemas Complejos, Universidad de Zaragoza, Mariano Esquillor s/n, 50018 Zaragoza, Spain \\ ${ }^{3}$ Unidad Asociada IQFR-BIFI, Universidad de Zaragoza, Mariano Esquillor s/n, 50018 Zaragoza, Spain \\ ${ }^{4}$ Fundación ARAID, Universidad de Zaragoza, Mariano Esquillor s/n, 50018 Zaragoza, Spain \\ ${ }^{5}$ Departament d'Enginyeria Agroforestal, Escola Tècnica Superior d'Enginyeria Agrària, Universitat de Lleida, \\ Alcalde Rovira Roure 191, 25198 Lleida, Spain \\ ${ }^{6}$ Instituto de Química-Física Rocasolano, Consejo Superior de Investigaciones Científicas, Serrano 119, 28006 Madrid, Spain
}

(Received 24 July 2014; published 25 February 2015)

\begin{abstract}
Schrödinger suggested that thermodynamical functions cannot be based on the gratuitous allegation that quantum-mechanical levels (typically the orthogonal eigenstates of the Hamiltonian operator) are the only allowed states for a quantum system [E. Schrödinger, Statistical Thermodynamics (Courier Dover, Mineola, 1967)]. Different authors have interpreted this statement by introducing density distributions on the space of quantum pure states with weights obtained as functions of the expectation value of the Hamiltonian of the system. In this work we focus on one of the best known of these distributions and prove that, when considered in composite quantum systems, it defines partition functions that do not factorize as products of partition functions of the noninteracting subsystems, even in the thermodynamical regime. This implies that it is not possible to define extensive thermodynamical magnitudes such as the free energy, the internal energy, or the thermodynamic entropy by using these models. Therefore, we conclude that this distribution inspired by Schrödinger's idea cannot be used to construct an appropriate quantum equilibrium thermodynamics.
\end{abstract}

DOI: 10.1103/PhysRevE.91.022137

PACS number(s): 05.30.Ch, 05.70.-a, 03.65.Aa

\section{INTRODUCTION}

In a note to the second edition of his book on statistical thermodynamics [1], Schrödinger suggests that thermodynamical functions cannot be based on the gratuitous allegation that quantum-mechanical levels (typically the orthogonal eigenstates of the Hamiltonian operator) are the only allowed states in statistical thermodynamics. In Khinchin's classical book on the mathematical foundations of quantum statistics [2], an approach to microcanonical averages is proposed in line with Schrödinger's suggestion. Landau and Lifshitz [3] also consider unrealistic the possibility of preparing isolated macroscopic physical systems in a precise energy eigenstate since energy levels are too close to each other to select just one.

From these points of view, it seems then natural to consider the description of quantum-statistical systems in terms of probability densities defined on the space of physical states. This description offers the possibility of considering the weight of a given state in an analogous manner to what it is done when describing classical statistical systems. The approach also offers many advantages in the description of hybrid quantumclassical systems, as it can be seen in [4,5]. Nonetheless, it is important to keep in mind that these distributions are ambiguous from the physical point of view. Indeed, it is well known since von Neumann [6] that the physical properties of a quantum system are encoded univocally in its density matrix $\hat{\rho}$, but there are many different equivalent expressions for $\hat{\rho}$ as a sum of pure states (projectors). Therefore, we may define entirely different distributions on the space of states, which nevertheless are equivalent because they lead to the same density matrix.

In Refs. [7-9] Brody and co-workers proposed their quantum microcanonical postulate, which asserts that every quantum state possessing the same energy expectation value must be realized with the same probability. Brody and coworkers also introduced an alternative quantum canonical distribution and studied some of its properties. The distribution is defined as a density on the space of pure quantum states that assigns to each state the Boltzmann weight associated with the expectation value of the Hamiltonian. These ideas have been adopted with different degrees of intensity by several authors using even different distributions (see Refs. [10-22] for some examples). In this work we focus on the particular state-space distribution introduced in Refs. [7-11] and, following JonaLasinio and Presilla, we refer to it as the Schrödinger-Gibbs (SG) distribution. A crucial result of this construction is that, as suggested by Fig. 2 of Ref. [7], the third law of thermodynamics may not be satisfied. Therefore, it is desirable to know why this happens and whether some other essential properties of the equilibrium thermodynamics, such as the definition of consistent extensive magnitudes, are maintained when using the SG distribution.

In this work we prove that, when considered on composite noninteracting quantum systems, this distribution defines partition functions that do not factorize as products of partition functions of the subsystems, even in the thermodynamical regime. This implies that it is not possible to define extensive thermodynamical magnitudes such as the free energy, the internal energy, or the thermodynamic entropy by using these models. Therefore, although Schrödinger's suggestion might seem reasonable a priori and it could have interesting dynamical features (as can be learned from some of the references above), it cannot be used to construct an appropriate quantum thermodynamics, at least not if the suggestion is materialized in the form of the SG distribution used as an equilibrium distribution. 
The structure of the paper is as follows. In Sec. II A we review the traditional canonical distribution as well as the SG distribution and we discuss the different alternative forms to present them. Section II B studies the quantum properties associated with the density matrix that encodes the properties of the SG distribution, in particular that it commutes with the Hamiltonian. Sections IIC and IID contain the main result of the paper: We prove that the partition function of the $S G$ quantum ensemble does not factorize in the case of composite noninteracting systems. In Sec. III we present a simple model to exemplify our analysis and discuss it from several points of view, namely, we evaluate the partition function, the possible definitions of thermodynamic extensive magnitudes, and an alternative description in terms of density matrices. In both approaches we identify the lack of factorizability and one of its causes: basically, that the density matrix associated with the SG distribution represents an entangled state. In Sec. IV we briefly summarize the content of the paper and consider the potential impact of our analysis on the study of the equilibrium and nonequilibrium statistics of hybrid quantum-classical systems.

\section{TRADITIONAL AND SG QUANTUM CANONICAL ENSEMBLES}

\section{A. Description of the ensembles}

Consider a physical system on a Hilbert space $\mathcal{H}$ of dimension $n$. We define the dynamics by a Hamiltonian $\hat{H}$. In this context, the traditional quantum canonical ensemble (QCE) (see standard textbooks such as Refs. [6,23-25] for details) is the ensemble defined by the density matrix

$$
\hat{\rho}_{C}=Z^{-1} \exp (-\beta \hat{H}),
$$

where the partition function $Z$ is defined as

$$
Z=\operatorname{Tr}[\exp (-\beta \hat{H})]
$$

One may alternatively arrive at ensemble definitions by establishing first a probability distribution $F(|\psi\rangle)$ on the Hilbert space $\mathcal{H}$ with respect to the canonical volume element $d \mu(|\psi\rangle)$ or, equivalently, a probability distribution $F\left(P_{\psi}\right)$ on the projective space $\mathcal{P} \mathcal{H}$, where $P_{\psi}$ is the orthogonal projector onto the one-dimensional subspace generated by state $\psi$. These distributions can then be used to define the ensembles, i.e., to construct the density matrices: Given any probability distribution $F$ defined on the state space, the corresponding density matrix is given by

$$
\hat{\rho}=\int_{\mathcal{P H}} d \mu\left(P_{\psi}\right) F\left(P_{\psi}\right) P_{\psi}
$$

where $d \mu$ represents a (dynamically) invariant measure on $\mathcal{P H}$. Different distributions $F$ and $F^{\prime}$ may lead to the same density matrix and must then be considered equivalent. This is just reflecting the well known ambiguity in the definition of a density matrix: Given a density matrix $\hat{\rho}$, there are infinitely many ways of writing it as a convex combination of rank-one projectors.
One may now prove that the average value of an arbitrary quantum observable $\hat{O}$ can be calculated as (see [5])

$$
\langle\hat{O}\rangle=\int_{\mathcal{P H}} d \mu\left(P_{\psi}\right) F\left(P_{\psi}\right) O\left(P_{\psi}\right),
$$

where $O\left(P_{\psi}\right)=\operatorname{Tr}\left(\hat{O} P_{\psi}\right)=\frac{\langle\psi \mid \hat{O} \psi\rangle}{\langle\psi \mid \psi\rangle}$. However, the result is exactly the same if we compute

$$
\langle\hat{O}\rangle=\operatorname{Tr}(\hat{\rho} \hat{O})
$$

where $\hat{\rho}$ is Eq. (2.3).

One can construct one such probability distribution to obtain the quantum canonical ensemble $\hat{\rho}_{C}$. Let us begin with a distribution defined on the Hilbert space as

$$
\begin{aligned}
F(|\psi\rangle) & =Z^{-1} \sum_{k} e^{-\beta E_{k}} \delta\left(|\psi\rangle-\left|E_{k}\right\rangle\right) \\
& =-\sum_{k} \beta^{-1} \frac{\partial \ln Z}{\partial E_{k}} \delta\left(|\psi\rangle-\left|E_{k}\right\rangle\right),
\end{aligned}
$$

with the partition function

$$
Z=\int_{\mathcal{H}} d \mu(|\psi\rangle) \sum_{k} e^{-\beta E_{k}} \delta\left(|\psi\rangle-\left|E_{k}\right\rangle\right)=\sum_{k} e^{-\beta E_{k}}
$$

where $\left\{\left|E_{k}\right\rangle\right\}_{k=1, \ldots, n}$ represent the energy eigenvectors of the Hamiltonian $\hat{H}$ and $\left\{E_{k}\right\}$ the corresponding eigenvalues. Of course, as we mentioned above, this representation is not unique.

If we prefer to consider the distribution as defined on the projective space $\mathcal{P} \mathcal{H}$, we have

$$
F\left(P_{\psi}\right)=Z^{-1} \sum_{k} e^{-\beta E_{k}} \delta\left(P_{\psi}-\frac{\left|E_{k}\right\rangle\left\langle E_{k}\right|}{\left\langle E_{k} \mid E_{k}\right\rangle}\right),
$$

where now the partition function is written as

$$
Z=\int_{\mathcal{P} \mathcal{H}} d \mu\left(P_{\psi}\right) \sum_{k} e^{-\beta E_{k}} \delta\left(P_{\psi}-\frac{\left|E_{k}\right\rangle\left\langle E_{k}\right|}{\left\langle E_{k} \mid E_{k}\right\rangle}\right)=\sum_{k} e^{-\beta E_{k}},
$$

where $d \mu$ represents an invariant measure on $\mathcal{P} \mathcal{H}$. Notice that these are just the expressions for the spectral decomposition of the density matrix (2.1) written as a probability distribution on $\mathcal{H}$ or $\mathcal{P} \mathcal{H}$. These distributions select, out of all states of $\mathcal{H}$ (equivalently of $\mathcal{P} \mathcal{H}$ ), only those that are eigenstates of the Hamiltonian and assign to them the corresponding Boltzmann probability. This may lead one to think that the canonical ensemble contains some kind of preference for those states, but this is not true. Invoking the above-mentioned nonuniqueness, one may use a different distribution $F$ to construct the canonical ensemble $\hat{\rho}_{C}$, without using $\delta$ functions centered at the Hamiltonian eigenstates. It could be a continuous distribution over the entire space or even one distribution that assigns zero probability to those eigenstates.

Brody and co-workers (see Refs. [7,9]) and Jona-Lasinio and Presilla (see Refs. [10,11]) introduced another distribution, following the seminal idea by Schrödinger [1]. The main point is to consider, instead of only one state for each eigenvalue of the Hamiltonian, all physical states of $\mathcal{H}$ (or $\mathcal{P} \mathcal{H}$ ) leading to the same expectation value for $\hat{H}$ and assign to all of them the Boltzmann probability with respect to that expectation value, 
i.e., $(1 / Z) \exp (-\beta\langle\psi|\hat{H}| \psi\rangle)$. As we said, we will call this distribution the Schrödinger-Gibbs distribution and represent it as $F^{\mathrm{SG}}$. Notice that, by construction, $F^{\mathrm{SG}}$ can be thought of as a direct generalization to the quantum realm of the classical canonical distribution. However, as we are going to see, its properties are different from the classical case because of the quantum nature of the states it is defined on.

The SG distribution on the Hilbert space can thus be written as

$$
F^{\mathrm{SG}}(|\psi\rangle)=\left(Z^{\mathrm{SG}}\right)^{-1} \delta(1-\langle\psi \mid \psi\rangle) e^{-\beta\langle\psi \mid \hat{H} \psi\rangle},
$$

where now the partition function reads

$$
\begin{aligned}
Z^{\mathrm{SG}} & =\int_{\mathcal{H}} d \mu(|\psi\rangle) \delta(1-\langle\psi \mid \psi\rangle) e^{-\beta\langle\psi \mid \hat{H} \psi\rangle} \\
& =\int_{S} d \mu(|\psi\rangle) e^{-\beta\langle\psi \mid \hat{H} \psi\rangle}
\end{aligned}
$$

where we denote by $S$ the sphere of vectors in $\mathcal{H}$ of norm equal to one [26]. This last distribution is equivalent to a distribution $F^{\mathrm{SG}}$ defined on the projective space $\mathcal{P} \mathcal{H}$ modulo a constant factor that does not affect the resulting ensemble (if interested see Appendix B to understand the origin of the constant factor). If we define the expectation value of the operator $\hat{H}$ on projector $P_{\psi}=\frac{|\psi\rangle\langle\psi|}{\langle\psi \mid \psi\rangle} \in \mathcal{P} \mathcal{H}$ as

$$
H\left(P_{\psi}\right):=\operatorname{Tr}\left(P_{\psi} \hat{H}\right),
$$

the distribution can be written as

$$
F^{\mathrm{SG}}\left(P_{\psi}\right)=\left(Z^{\mathrm{SG}}\right)^{-1} e^{-\beta H\left(P_{\psi}\right)},
$$

with the partition function

$$
Z^{\mathrm{SG}}=\int_{\mathcal{P H}} d \mu\left(P_{\psi}\right) e^{-\beta H\left(P_{\psi}\right)} .
$$

If we evaluate the density matrix associated with the distribution (2.13) (and use the result proved in Appendix B to evaluate the integrals on the sphere $S$ of vectors of $\mathcal{H}$ with norm equal to one) we obtain

$$
\hat{\rho}^{\mathrm{SG}}=\left(Z^{\mathrm{SG}}\right)^{-1} \int_{S} d \mu(|\psi\rangle) e^{-\beta\langle\psi \mid \hat{H} \psi\rangle}|\psi\rangle\langle\psi| .
$$

Regarding the measure $d \mu$, a few comments are in order. It is well known (see [4,5] and references therein) that finite-dimensional quantum systems defined on $\mathbb{C}^{n}$ admit a Hamiltonian structure associated with the canonical Kähler structure of the manifold. The Schrödinger equation corresponds then to the flow of a Hamiltonian vector field associated with the canonical symplectic form. Therefore, the Liouville theorem ensures that the corresponding symplectic volume is preserved by the dynamics, as it happens in classical mechanics. As the sphere of vectors with norm one is also preserved by the dynamics, the natural choice for $d \mu$ on $S$ is the restriction of the symplectic volume of $\mathbb{C}^{n}$. Regarding the projective space $\mathcal{P} \mathcal{H}$, again there exists a canonical Kähler structure on it, also preserved by the dynamics. Therefore, we can also consider the corresponding symplectic volume, which will also be invariant.

Now we ask what the physical origin of the differences between Eqs. (2.1) and (2.15) is. If one approaches the canonical ensemble from the microcanonical one, the origin is clear. Indeed, while the microcanonical ensemble in Refs. [7-9] is based on the postulate that every state possessing the same energy expectation value must be democratically realized (which implements Schrödinger's suggestion), in the traditional microcanonical ensemble, orthogonal states must be the only ones to be included in the sample space of the statistical thermodynamics. The arguments for using only orthogonal states are based on the standard theory of probability (see, for instance, Secs. 4.5 and 4.6 of Refs. [27] and [28]) and on the relationship between information theory and thermodynamics [29-37].

\section{B. Quantum properties of the Schrödinger-Gibbs distribution}

Let us now study the properties of the density matrix (2.15) in more detail. First of all, for technical reasons that will become clear later, we want to prove that the SG density matrix commutes with the Hamiltonian and hence both operators can be diagonalized in a common eigenbasis. Notice that if we consider $\hat{\rho}^{\mathrm{SG}}$ as a stationary distribution corresponding to an equilibrium situation and the Hamiltonian does not depend on time, the result is trivial:

$i \hbar \frac{\partial \hat{\rho}^{\mathrm{SG}}(t)}{\partial t}=\left[\hat{H}, \hat{\rho}^{\mathrm{SG}}(t)\right] \rightarrow \frac{\partial \hat{\rho}^{\mathrm{SG}}(t)}{\partial t}=0 \Rightarrow\left[\hat{H}, \hat{\rho}^{\mathrm{SG}}(t)\right]=0$.

However, a priori there is no reason to consider $\hat{\rho}^{\mathrm{SG}}$ to be stationary. In the following we will prove that it is indeed time independent. The simplest way to do this is to consider a unitary evolution for $\hat{\rho}^{\mathrm{SG}}$ of the form

$$
\hat{\rho}^{\mathrm{SG}}(t)=\hat{U}(t) \hat{\rho}^{\mathrm{SG}}(0) \hat{U}^{\dagger}(t),
$$

where, as we assume that the Hamiltonian does not depend on time,

$$
\hat{U}(t):=e^{-i \hat{H} t} .
$$

Under the same evolution, the volume element $d \mu$ on $S$ and the expectation value of the energy $\langle\psi \mid \hat{H} \psi\rangle$ are, because of unitarity, constant in time. Thus, for any $t$ we can write

$$
\langle\psi(t) \mid \hat{H} \psi(t)\rangle=\langle\psi(0) \mid \hat{H} \psi(0)\rangle \quad \forall t
$$

and thus, as the volume element is also preserved, we find

$$
\begin{aligned}
& d \mu[|\psi(0)\rangle]\left(Z^{\mathrm{SG}}\right)^{-1} e^{-\beta\langle\psi(0) \mid \hat{H} \psi(0)\rangle} \\
& \quad=d \mu[|\psi(t)\rangle]\left(Z^{\mathrm{SG}}\right)^{-1} e^{-\beta\langle\psi(t) \mid \hat{H} \psi(t)\rangle} \quad \forall t .
\end{aligned}
$$

Consider now the expression of the density matrix $\hat{\rho}^{\mathrm{SG}}$ defined in Eq. (2.15), which we denote by $\hat{\rho}_{0}^{\text {SG }}$. Let us consider how the unitary evolution transforms this density matrix after an arbitrary but fixed value of time $t_{*}$, i.e.,

$$
\begin{aligned}
\hat{\rho}^{\mathrm{SG}}\left(t_{*}\right) & =\hat{U}\left(t_{*}\right) \hat{\rho}_{0}^{\mathrm{SG}} \hat{U}^{\dagger}\left(t_{*}\right) \\
& =\left(Z^{\mathrm{SG}}\right)^{-1} \int_{S} d \mu(|\psi\rangle) e^{-\beta\langle\psi \mid \hat{H} \psi\rangle} \hat{U}\left(t_{*}\right)|\psi\rangle\langle\psi| \hat{U}\left(t_{*}\right)^{\dagger}
\end{aligned}
$$

Here $\hat{U}\left(t_{*}\right)$ defines a transformation on the set of states as

$$
\left|\psi^{\prime}\right\rangle=\hat{U}\left(t_{*}\right)|\psi\rangle
$$


which obviously preserves the sphere $S$ since $\langle\psi \mid \psi\rangle=\left\langle\psi^{\prime} \mid \psi^{\prime}\right\rangle$ and thus we can rewrite Eq. (2.19) as

$$
\hat{\rho}^{\mathrm{SG}}\left(t_{*}\right)=\left(Z^{\mathrm{SG}}\right)^{-1} \int_{S} d \mu(|\psi\rangle) e^{-\beta\langle\psi \mid \hat{H} \psi\rangle}\left|\psi^{\prime}\right\rangle\left\langle\psi^{\prime}\right| .
$$

We know that the measure is invariant, i.e.,

$$
d \mu(|\psi\rangle)\left(Z^{\mathrm{SG}}\right)^{-1} e^{-\beta\langle\psi \mid \hat{H} \psi\rangle}=d \mu\left(\left|\psi^{\prime}\right\rangle\right)\left(Z^{\mathrm{SG}}\right)^{-1} e^{-\beta\left\langle\psi^{\prime} \mid \hat{H} \psi^{\prime}\right\rangle},
$$

and therefore, as the sphere $S$ is invariant and the integral runs over all normalized states, we can write

$$
\hat{\rho}^{\mathrm{SG}}\left(t_{*}\right)=\left(Z^{\mathrm{SG}}\right)^{-1} \int_{S} d \mu\left(\left|\psi^{\prime}\right\rangle\right) e^{-\beta\left\langle\psi^{\prime} \mid \hat{H} \psi^{\prime}\right\rangle}\left|\psi^{\prime}\right\rangle\left\langle\psi^{\prime}\right|,
$$

which is the same as Eq. (2.15). Hence, we have proved that

$$
\hat{\rho}^{\mathrm{SG}}\left(t_{*}\right)=\hat{\rho}_{0}^{\mathrm{SG}} .
$$

As the value of $t_{*}$ is generic, we conclude that the density matrix is independent of $t$ and hence commutes with the Hamiltonian

$$
\left[\hat{H}, \hat{\rho}^{\mathrm{SG}}\right]=0 .
$$

On a different matter, in order to compute the integral in Eq. (2.15), we can use the tools of complex analysis, as presented in Appendix C. The result for the partition function is obtained in Eqs. (C9)-(C11). Assuming that there exist $p+1$ different eigenvalues of the Hamiltonian $\hat{H}$ with degeneracies $d_{0}, \ldots, d_{p}$, the partition function is written as

$$
Z^{\mathrm{SG}}=\sum_{k=0}^{p} e^{-\beta E_{k}} F_{E_{k}}
$$

where

$$
\begin{aligned}
F_{E_{k}}= & -\frac{(2 \pi)^{n}}{\left(d_{k}-1\right) !} \sum_{\substack{j_{0}, \ldots, j_{p}=0 \\
j_{0}+j_{1}+\cdots+j_{p}=d_{k}-1}}^{d_{k}-1}\left(\begin{array}{c}
d_{k}-1 \\
j_{0}, \ldots, j_{p}
\end{array}\right) \\
& \times \prod_{\substack{s=0 \\
s \neq k}}^{p} \frac{(-1)^{j_{0}}\left[\left(d_{s}+j_{s}-1\right) ! /\left(d_{s}-1\right) !\right]}{\left(\beta E_{s}-\beta E_{k}\right)^{d_{s}+j_{s}}},
\end{aligned}
$$

with $\left(\begin{array}{c}d_{k}-1 \\ j_{0}, \ldots, j_{s}\end{array}\right)$ representing the multinomial coefficient

$$
\left(\begin{array}{c}
d_{k}-1 \\
j_{0}, \ldots, j_{s}
\end{array}\right)=\frac{\left(d_{k}-1\right) !}{j_{0} ! \cdots j_{s} !} .
$$

Notice that the traditional distribution (2.6) associated with the QCE corresponds to the case where

$$
F_{E_{k}}=d_{k} \quad \forall k=0, \ldots, p,
$$

where $d_{k}$ stands for the degeneracy of the energy level $E_{k}$.

From the above expressions we can obtain the spectral decomposition of the density matrix $\hat{\rho}^{\mathrm{SG}}$ defined by Eq. (2.15). Indeed, we know that $\hat{\rho}^{\mathrm{SG}}$ is diagonal in the energy eigenbasis and thus it can be written as

$$
\hat{\rho}^{\mathrm{SG}}=\sum_{k} \omega_{k} \frac{\left|E_{k}\right\rangle\left\langle E_{k}\right|}{\left\langle E_{k} \mid E_{k}\right\rangle}
$$

where the eigenvalue $\omega_{k}$ corresponding to the eigenvector $\left|E_{k}\right\rangle$ is equal to

$$
\omega_{k}=\hat{\rho}_{k k}^{\mathrm{SG}}=\left(Z^{\mathrm{SG}}\right)^{-1} \int_{S} d \mu(|\psi\rangle) \exp \left(-\beta \sum_{j} E_{j}\left|\psi_{j}\right|^{2}\right)\left|\psi_{k}\right|^{2}
$$

and $|\psi\rangle=\sum_{j} \psi_{j}\left|E_{j}\right\rangle$. Each $\omega_{k}$ is thus obtained as a suitable derivative of the partition function $Z^{\mathrm{SG}}$, as in the case of the canonical distribution

$$
\begin{aligned}
\omega_{k} & =-\beta^{-1} \frac{\partial \ln Z^{\mathrm{SG}}}{\partial E_{k}} \\
& =\frac{e^{-\beta E_{k}} F_{E_{k}}}{Z^{\mathrm{SG}}}-\beta^{-1} \frac{\sum_{j=0}^{p} e^{-\beta E_{j}}\left(\partial F_{E_{j}} / \partial E_{k}\right)}{Z^{\mathrm{SG}}} .
\end{aligned}
$$

Therefore, we just proved that the probability distribution (2.10) can be written as

$$
\begin{aligned}
F^{\mathrm{SG}}(|\psi\rangle)= & \sum_{k}\left(\frac{e^{-\beta E_{k}} F_{E_{k}}}{Z^{\mathrm{SG}}}-\beta^{-1} \frac{\sum_{j=0}^{p} e^{-\beta E_{j}}\left(\partial F_{E_{j}} / \partial E_{k}\right)}{Z^{\mathrm{SG}}}\right) \\
& \times \delta\left(|\psi\rangle-\left|E_{k}\right\rangle\right),
\end{aligned}
$$

which is formally analogous to the distribution (2.6), but with very different content because of the second term in the sum. If we write it as a density matrix we have

$$
\begin{aligned}
\hat{\rho}^{\mathrm{SG}}= & \sum_{k}\left(\frac{e^{-\beta E_{k}} F_{E_{k}}}{Z^{\mathrm{SG}}}-\beta^{-1} \frac{\sum_{j=0}^{p} e^{-\beta E_{j}}\left(\partial F_{E_{j}} / \partial E_{k}\right)}{Z^{\mathrm{SG}}}\right) \\
& \times \frac{\left|E_{k}\right\rangle\left\langle E_{k}\right|}{\left\langle E_{k} \mid E_{k}\right\rangle} .
\end{aligned}
$$

Notice that the second term is such that $\sum_{k} \frac{\partial F_{E_{j}}}{\partial E_{k}}=0$ and then $\operatorname{Tr} \hat{\rho}^{\mathrm{SG}}=1$.

Only if the functions $F_{E_{k}}$ were independent from the energy eigenvalues and equal to the degeneracies $d_{k}$ would $\hat{\rho}^{\mathrm{SG}}$ coincide with the usual canonical distribution $\hat{\rho}_{C}$ in Eq. (2.1). Thus, given any $\beta$, the average value of any quantum observable $\hat{O}$ will be in general different in the ensembles and, in general, the properties of the ensembles will not coincide. We will return to this point in Sec. III.

A similar computation for some particular cases can be found in [38]. Indeed, the authors analyze the completely nondegenerate case of a general system nd the strong-coupling limit of a spin-spin interaction Hamiltonian. Nonetheless, the authors of [38] do not study the implications of their results at the thermodynamic level, as we will in the next section.

\section{Thermodynamical properties of the Schrödinger-Gibbs distribution}

We have just seen how, even if $F^{\mathrm{SG}}$ is analogous to the canonical distribution of a classical (continuous) system, the quantum nature of the system it describes makes it equivalent to a quantum ensemble described by a density matrix $\hat{\rho}^{\mathrm{SG}}$ that is different from the traditional canonical one. We want to check now if, despite these differences, we can still define an appropriate thermodynamics associated with the SG distribution. 
In order to define a system in thermodynamical equilibrium, we must obtain the corresponding thermodynamic magnitudes, both extensive and intensive ones, from the statistical-mechanics objects. Thus, for a system described by a partition function $Z$, the Helmholtz free energy $F$ (which is the natural free energy in the canonical ensemble) corresponds to

$$
F=-\beta^{-1} \ln Z,
$$

the thermodynamic (also called sometimes internal) energy $U$ corresponds to the average value of the energy, which can be obtained through

$$
U=-\frac{\partial \ln Z}{\partial \beta},
$$

and the thermodynamic entropy $S^{\text {th }}$ is written as

$$
S^{\text {th }}=k_{B}\left(\ln Z-\beta \frac{\partial \ln Z}{\partial \beta}\right) .
$$

If the partition function $Z$ is factorizable, the functions $F, U$, and $S^{\text {th }}$ are extensive magnitudes. Then the extensiveness of the functions $F, U$, or $S^{\text {th }}$ in the present case is equivalent to the factorizability property of the partition function $Z^{\mathrm{SG}}$. That is, if we consider a system defined as the composition of noninteracting subsystems, the partition function of the complete system must be written as the product of the partition function restricted to the subsystems in order to yield extensive thermodynamic functions. Notice that, so far, $\beta$ represents just a parameter that is used to define the partition function and ultimately may not have any physical meaning (for example, if the resulting thermodynamics is found to be inappropriate).

Consider then that the total Hilbert space $\mathcal{H}$ is equal to the tensor product of the Hilbert spaces describing the corresponding subsystems, i.e.,

$$
\mathcal{H}=\mathcal{H}_{1} \otimes \cdots \otimes \mathcal{H}_{m},
$$

with dimensions $n_{1}, n_{2}, \ldots, n_{m}$ (for simplicity we assume the case of finite-dimensional quantum systems). The dimension of $\mathcal{H}$ is thus given by $n=n_{1}, \ldots, n_{m}$. Since we assume that there is no interaction among the subsystems, the total Hamiltonian is written as the sum

$\hat{H}=\sum_{\alpha=1}^{m} \hat{H}_{\alpha}=\sum_{\alpha=1}^{m} \hat{\mathbb{I}}_{n_{1}} \otimes \cdots \otimes \hat{\mathbb{I}}_{n_{\alpha-1}} \otimes \hat{h}_{\alpha} \otimes \hat{\mathbb{I}}_{n_{\alpha+1}} \otimes \cdots \otimes \hat{\mathbb{I}}_{n_{m}}$.

As all $\hat{H}_{\alpha}$ commute, the tensor product of the eigenbases of the $\hat{h}_{\alpha}$ is an eigenbasis of the total Hamiltonian $\hat{H}$. We thus have that each element of the total-energy eigenbasis $\left\{\left|E_{k}\right\rangle\right\}$ (with corresponding eigenvalues $E_{k}$ ) can be written as the tensor product of the corresponding elements of the different eigenbases $\left\{\left|e_{j_{\alpha}}^{\alpha}\right\rangle\right\}$ of the subsystems (with corresponding eigenvalues $e_{k}^{\alpha}$ )

$$
\left|E_{k}\right\rangle=\left|e_{j_{1}}^{1}\right\rangle \otimes \cdots \otimes\left|e_{j_{m}}^{m}\right\rangle, \quad E_{k}=e_{j_{1}}^{1}+\cdots+e_{j_{m}}^{m} .
$$

Without loss of generality, we assume that

$$
E_{k} \leqslant E_{k+1}, \quad e_{j_{\alpha}}^{\alpha} \leqslant e_{j_{\alpha}+1}^{\alpha} \quad \forall \alpha .
$$

It is well known that the partition function of the canonical distribution $\hat{\rho}_{C}$ in Eq. (2.1) factorizes as a product of the partition function of the subsystems (see [25], Sec. 16.2). If we compute the partition function $Z^{\mathrm{SG}}$ in this basis, we obtain as a result Eq. (2.26). If we did the same computation restricted to the subsystem $\alpha$, we would obtain similar expressions for the partition functions $Z_{\alpha}^{\mathrm{SG}}$, with the eigenvalues $E_{k}$ replaced by the eigenvalues $e_{j_{\alpha}}^{\alpha}$. In order to check whether or not $Z^{\mathrm{SG}}$ factorizes, let us consider the limit $\beta \gg 1$, where we can write that

$$
\left.Z^{\mathrm{SG}}\right|_{\beta \gg 1} \sim e^{-\beta E_{0}} F_{E_{0}}
$$

Analogously,

$$
\left.Z_{\alpha}^{\mathrm{SG}}\right|_{\beta \gg 1} \sim e^{-\beta e_{0}^{\alpha}} F_{e_{0}^{\alpha}} .
$$

Factorizability of the partition function would require that

$$
\left.Z^{\mathrm{SG}}\right|_{\beta \gg 1}=\left.\prod_{\alpha=1}^{m} Z_{\alpha}^{\mathrm{SG}}\right|_{\beta \gg 1},
$$

which implies

$$
F_{E_{0}}=\prod_{\alpha=1}^{m} F_{e_{0}^{\alpha}} .
$$

However, we can immediately check that [39]

$$
F_{E_{0}} \neq \prod_{\alpha=1}^{m} F_{e_{0}^{\alpha}} .
$$

Indeed, in the nondegenerate ground-state case, Eq. (2.27) becomes for $E_{k}=E_{0}$ and $E_{k}=e_{0}^{\alpha}$, respectively,

$$
F_{E_{0}}=\prod_{j \neq 0} \frac{1}{\beta E_{j}-\beta E_{0}}, \quad F_{e_{0}^{\alpha}}=\prod_{j_{\alpha} \neq 0} \frac{1}{\beta e_{j_{\alpha}}^{\alpha}-\beta e_{0}^{\alpha}}
$$

and thus it is simple to prove that

$$
\prod_{j \neq 0} \frac{\beta^{-1}}{E_{j}-E_{0}} \neq \prod_{\alpha} \prod_{j_{\alpha} \neq 0} \frac{\beta^{-1}}{e_{j_{\alpha}}^{\alpha}-e_{0}^{\alpha}} .
$$

The last inequality can be easily understood by thinking that, on the right-hand side, there are only energy differences corresponding to a change in one subsystem, while on the left hand side all possible energy differences are considered.

This being so in the limit $\beta \gg 1$ is sufficient to prove that the partition function $Z^{\mathrm{SG}}$ is not the product of the partition functions of the subsystems and therefore we cannot define extensive magnitudes from $Z^{\mathrm{SG}}$. Besides, this property does not depend on the number of subsystems and therefore it is still valid in a thermodynamic limit where $m \rightarrow \infty$, i.e., we can claim that

$$
Z^{\mathrm{SG}} \neq \prod_{\alpha=1}^{\infty} Z_{\alpha}^{\mathrm{SG}} .
$$

We thus conclude that the thermodynamic functions defined by Eqs. (2.34), (2.35), or (2.36) cannot represent extensive magnitudes.

In principle we may study the same problem from the point of view of the density matrix $\hat{\rho}^{\mathrm{SG}}$, but doing so in full generality becomes quite difficult from the computational point of view. We will tackle this analysis in Sec. III for the case of a specific quantum system resulting from the composition of two-level 
systems and we will recover the same results obtained here but in a much simpler way.

\section{Origin of nonextensiveness: Integrating over entangled states}

Let us consider the simplest case of a composite system by assuming that we have $m=2$ in Eqs. (2.37) and (2.38). We know from Eq. (2.48) that

$$
Z_{1}^{\mathrm{SG}} Z_{2}^{\mathrm{SG}} \neq Z_{12}^{\mathrm{SG}} \text {. }
$$

Now we want to understand the origin of this difference from a physical point of view.

The partition functions for the individual subsystems and for the composite system will read, respectively,

$$
\begin{aligned}
Z_{1}^{\mathrm{SG}} & =\int_{\mathcal{S}^{n_{1}}} d \mu\left(\left|\psi^{1}\right\rangle\right) e^{-\beta\left\langle\psi^{1} \mid \hat{H}_{1} \psi^{1}\right\rangle}, \\
Z_{2}^{\mathrm{SG}} & =\int_{\mathcal{S}^{n_{2}}} d \mu\left(\left|\psi^{2}\right\rangle\right) e^{-\beta\left\langle\psi^{2} \mid \hat{H}_{2} \psi^{2}\right\rangle},
\end{aligned}
$$

and

$$
Z_{12}^{\mathrm{SG}}=\int_{\mathcal{S}^{n_{1} n_{2}}} d \mu(|\psi\rangle) e^{-\beta\langle\psi \mid \hat{H} \psi\rangle},
$$

where $\mathcal{S}^{n_{1}}, \mathcal{S}^{n_{2}}$, and $\mathcal{S}^{n_{1} n_{2}}$ represent the $\left(n_{1}-1\right)$-dimensional, $\left(n_{2}-1\right)$-dimensional, and $\left(n_{1} n_{2}-1\right)$-dimensional spheres. We can write the product of two integrals like that of Eq. (2.50) as

$$
\begin{aligned}
\left(Z_{1}^{\mathrm{SG}}\right)\left(Z_{2}^{\mathrm{SG}}\right)= & \int_{\mathcal{S}^{n_{1}} \times \mathcal{S}^{n_{2}}} d \mu_{1}\left(\left|\psi^{1}\right\rangle\right) d \mu_{2}\left(\left|\psi^{2}\right\rangle\right) e^{-\beta\left\langle\psi^{1} \mid \hat{H}_{1} \psi^{1}\right\rangle} \\
& \times e^{-\beta\left\langle\psi^{2} \mid \hat{H}_{2} \psi^{2}\right\rangle} .
\end{aligned}
$$

In a basis like the one in (2.39), constituted by separable vectors, we have

$$
\begin{aligned}
& e^{-\beta\left\langle\psi^{1} \mid \hat{H}_{1} \psi^{1}\right\rangle} e^{-\beta\left\langle\psi^{2} \mid \hat{H}_{2} \psi^{2}\right\rangle} \\
& \quad=\exp \left(-\beta \sum_{k=1}^{n_{1}} e_{k}^{1}\left|\psi_{k}^{1}\right|^{2}\right) \exp \left(-\beta \sum_{j=1}^{n_{2}} e_{j}^{2}\left|\psi_{j}^{2}\right|^{2}\right) .
\end{aligned}
$$

The separability of the vectors allows us to write

$$
\sum_{k=1}^{n_{1}} e_{k}^{1}\left|\psi_{k}^{1}\right|^{2}+\sum_{j=1}^{n_{2}} e_{j}^{2}\left|\psi_{j}^{2}\right|^{2}=\langle\psi \mid \hat{H} \psi\rangle, \quad \psi=\psi^{1} \otimes \psi^{2} .
$$

Thus the resulting exponent is the expectation value of the Hamiltonian $\hat{H}=\hat{H}_{1} \otimes \mathbb{I}_{2}+\mathbb{I}_{1} \otimes \hat{H}_{2}$, evaluated at the separable points of $\mathcal{H}=\mathcal{H}_{1} \otimes \mathcal{H}_{2}$. Notice that in the sum above, $k$ runs from 1 to $n_{1}$ and $j$ from 1 to $n_{2}$ in such a way that the coordinate expression of a separable state $\psi$ reads

$$
\psi=\psi^{1} \otimes \psi^{2}=\left(\sum_{k} \psi_{k}^{1}\left|e_{k}^{1}\right\rangle\right) \otimes\left(\sum_{j} \psi_{j}^{2}\left|e_{j}^{2}\right\rangle\right) .
$$

Putting everything together, we have just proved that

$$
\left(Z_{1}^{\mathrm{SG}}\right)\left(Z_{2}^{\mathrm{SG}}\right)=\int_{\mathcal{S}^{n_{1}} \times \mathcal{S}^{n_{2}}} d \mu_{1}\left(\left|\psi^{1}\right\rangle\right) d \mu_{2}\left(\left|\psi^{2}\right\rangle\right) e^{-\beta\langle\psi \mid \hat{H} \psi\rangle} .
$$

Equation (2.57) sums the same function as (2.52), but only over the separable states of $\mathcal{S}^{n_{1} n_{2}} \subset \mathcal{H}=\mathcal{H}_{1} \otimes \mathcal{H}_{2}$, while (2.52) integrates over all states, both separable and entangled. This explains Eq. (2.49) from the physical point of view.

If we compare this situation with the traditional canonical distribution described by Eq. (2.6), we see that the $\delta$ functions would restrict the corresponding integrals to the eigenvectors of the Hamiltonian $\hat{H}$, which, in the case of noninteracting subsystems, correspond always to separable states. In addition, the statistical weight assigned to each of these eigenstates depends only on the eigenspace. In contrast, if we think about the SG distribution in terms of the corresponding density matrix [i.e., using expression (2.33)], we would be considering, apparently, only separable states (the Hamiltonian eigenstates participating of the spectral decomposition), but now the statistical weights associated with them do not factorize. Therefore, the density matrix (2.33) is not separable. We will illustrate this in a simple example in next section.

\section{A SIMPLE EXAMPLE}

In this section we present numerical examples of the properties discussed in the previous section. We will analyze the two different distributions (2.6) and (2.11) for a system of $N$ noninteracting two-level particles whose dynamics is described by a Hamiltonian of the form

$$
\hat{H}=\sum_{k} \hat{H}_{k}=\sum_{k} \overbrace{\hat{\mathbb{I}}_{2} \otimes \cdots \otimes \hat{\mathbb{I}}_{2}}^{k-1} \otimes \hat{h}_{k} \otimes \overbrace{\hat{\mathbb{I}}_{2} \otimes \cdots \otimes \hat{\mathbb{I}}_{2}}^{N-k},
$$

where $\hat{\mathbb{I}}_{2}$ stands for the identity operator in two dimensions and the one-particle Hamiltonian $\hat{h}_{k}$ can be written in the corresponding eigenbasis as

$$
h_{k}=\left(\begin{array}{cc}
0 & 0 \\
0 & \Delta
\end{array}\right) \quad \forall k=1, \ldots, N,
$$

where $\Delta$ represents the energy gap.

Let us now consider a basis for $\mathcal{H}$ defined as the tensor product of the energy eigenbases of every two-level subsystem. Thus, if we write $n=2^{N}$ for short, we have the set of vectors of the form

$$
\left|i_{1}, \ldots, i_{n}\right\rangle=\left|i_{1}\right\rangle \otimes \cdots \otimes\left|i_{n}\right\rangle \quad \text { for }\left|i_{k}\right\rangle=|0\rangle,|1\rangle .
$$

We also fix an ordering for the basis

$$
\begin{aligned}
\mathcal{B}= & \{|0,0, \ldots, 0,0\rangle,|0,0, \ldots, 0,1\rangle,|0,0, \ldots, 1,0\rangle, \ldots, \\
& |0,1, \ldots, 1,1\rangle,|1,1, \ldots, 1\rangle\} .
\end{aligned}
$$

In this basis it is a simple task to verify that the eigenvalues $\left\{E_{k}\right\}$ of the total Hamiltonian $\hat{H}$ and the corresponding degeneracies $d_{k}$ are given by

$$
E_{k}=k \Delta, \quad d_{k}=\left(\begin{array}{l}
N \\
k
\end{array}\right), \quad k=0, \ldots, N
$$

\section{A. Partition function and factorizability}

Our goal now is to obtain the expressions for the Schrödinger-Gibbs distribution corresponding to this 
Hamiltonian. We saw that the ensemble is defined by the partition function in (2.11). Thus, our goal is to write

$$
Z^{\mathrm{SG}}=\int_{\mathcal{S}^{N+1}-1} d \mu(\psi) e^{-\beta\langle\psi \mid \hat{H} \psi\rangle}
$$

for the particular case of the Hamiltonian in Eq. (3.1). Previously, from the analysis in Appendix C, the expression for $Z^{\mathrm{SG}}$ was written as in Eq. (2.26), where now $n=2^{N}$ and the spectrum is given by Eq. (3.5).

The simplest cases can be easily written. Indeed, we can consider the case $N=1$ (i.e., $n=2$ ) and write Eq. (2.26) as

$$
Z_{N=1}^{\mathrm{SG}}=\frac{(2 \pi)^{2}\left(1-e^{-\beta \Delta}\right)}{\beta \Delta} .
$$

Analogously, the next case is $N=2$ (or, equivalently, $n=4$ ):

$$
Z_{N=2}^{\mathrm{SG}}=\frac{(2 \pi)^{4}\left(1-2 \beta \Delta e^{-\beta \Delta}-e^{-2 \beta \Delta}\right)}{2 \beta^{3} \Delta^{3}} .
$$

We can immediately check that, as we proved in general, the partition function does not factorize, i.e.,

$$
Z_{N=2}^{\mathrm{SG}} \neq\left(Z_{N=1}^{\mathrm{SG}}\right)^{2} \text {. }
$$

\section{B. Thermodynamic entropies and specific heat}

We can also compute the corresponding entropy functions $S^{\text {th }}$ using Eq. (2.36). We obtain

$$
S_{N=1}^{\mathrm{th}}=\frac{k_{B}\left\{-\beta \Delta+e^{\beta \Delta}+\left(e^{\beta \Delta}-1\right) \ln \left[(2 \pi)^{2}\left(1-e^{-\beta \Delta}\right) / \beta \Delta\right]-1\right\}}{e^{\beta \Delta}-1}
$$

and

$$
\begin{aligned}
S_{N=2}^{\mathrm{th}}= & k_{B}\left(\frac{\left(-2 \beta \Delta e^{\beta \Delta}+e^{2 \beta \Delta}-1\right) \ln \left[8 \pi^{4} e^{-2 \beta \Delta}\left(-2 \beta \Delta e^{\beta \Delta}+e^{2 \beta \Delta}-1\right) / \beta^{3} \Delta^{3}\right]}{-2 \beta \Delta e^{\beta \Delta}+e^{2 \beta \Delta}-1}\right. \\
& \left.+\frac{-2 \beta \Delta-2 \beta \Delta e^{\beta \Delta}(\beta \Delta+2)+3 e^{2 \beta \Delta}-3}{-2 \beta \Delta e^{\beta \Delta}+e^{2 \beta \Delta}-1}\right) .
\end{aligned}
$$

Again, it is simple to verify that these functions are not additive, i.e., that

$$
S_{N=2}^{\text {th }} \neq 2 S_{N=1}^{\text {th }} \text {. }
$$

Therefore, we must conclude that $S^{\text {th }}$ cannot model an extensive magnitude and hence it cannot represent the thermodynamic entropy of a physical system. Besides this nonextensiveness, $S^{\text {th }}$ does not satisfy other properties that are required for the thermodynamic entropy, such as positiveness, and it must tend to zero in the limit where $T=\frac{1}{k_{B} \beta}$ tends to zero. Of course, in this case, $T$ must be considered just a parameter and in no way can it be identified with a physical temperature. Notice that this behavior does not follow from the nonextensiveness of the ensemble since it is present in the $N=1$ case where nonextensiveness is meaningless. As we mentioned in the previous section, the ensemble encoded in Eq. (2.33) is completely different from the canonical ensemble (2.1) and therefore its properties will, in general, be different. Thus it is natural that $\hat{\rho}^{C}$ defines extensive thermodynamical functions while $\hat{\rho}^{\mathrm{SG}}$ does not and it is also natural that the behaviors in the limit $T \rightarrow 0$ of the corresponding entropies differ. Regarding the nonextensiveness of $\hat{\rho}^{\mathrm{SG}}$, we proved in Sec. II D that it is related to the integration over entangled states in the definition of the ensemble (2.14). We do not have a similar proof to explain the behavior of the entropy in the $T \rightarrow 0$ limit, but it is evident that entanglement is not the only reason.

If we also compute the case $N=3$ (see [40]), we can represent the corresponding entropies together with the previous ones and confirm that, again, their zero- $T$ limit is different from zero and the functions are negative in a measurable part of their domains (see Fig. 1). If we consider the analogous quantity for the canonical ensemble [ $\hat{\rho}^{C}$ defined in Eq. (2.1)] in the same system, we find a very different behavior. Indeed, we can immediately obtain that

$$
S_{N}^{c, \text { th }}=k_{B}\left(\frac{N \beta \Delta e^{-\beta \Delta}}{e^{-\beta \Delta}+1}+\ln \left[\left(e^{-\beta \Delta}+1\right)^{N}\right]\right) .
$$

We can plot the first three cases for the same parameter values as before and obtain an equally spaced set of functions, as we can see in Fig. 2. We can also consider other magnitudes such as the specific heat $C_{v}$ (computed as $C_{v}=-\beta^{2} \frac{\partial U}{\partial \beta}$ ), which we plot in Fig. 3. For $C_{v}$ we recover the dependence obtained by Brody and co-workers in the simplest $N=1$ case [7], but we can also see (due to the more general calculations in this work) how $C_{v}$ scales with the number of subsystems $N$. Finally,

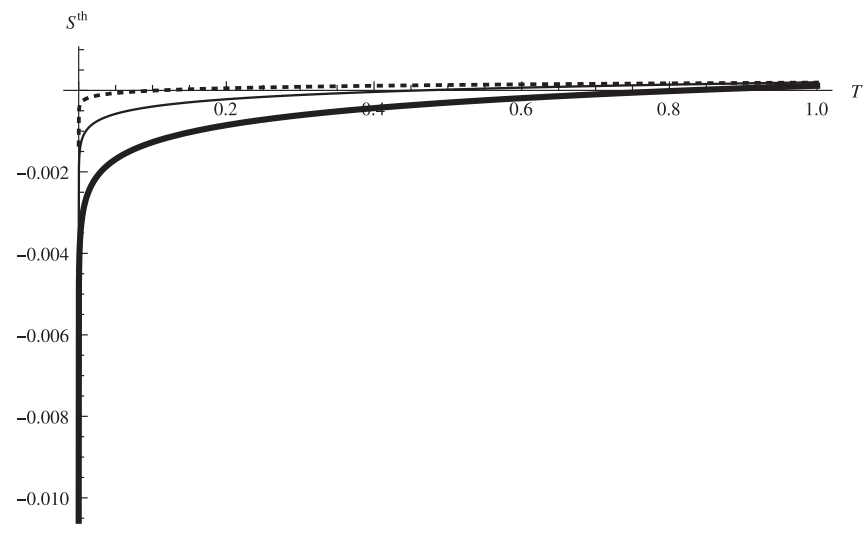

FIG. 1. Plot of the functions $S_{N=1}^{\text {th }}$ (dotted line), $S_{N=2}^{\text {th }}$ (thin line), and $S_{N=3}^{\text {th }}$ (thick line) versus $T$ for $k_{B}=8.617 \times 10^{-5}$ and $\Delta=0.001$ for the $\mathrm{SG}$ ensemble. We can easily verify the nonadditivity property of the function $S^{\text {th }}$ and the violation of the third law, since the entropy does not go to zero in the limit $T \rightarrow 0$. 


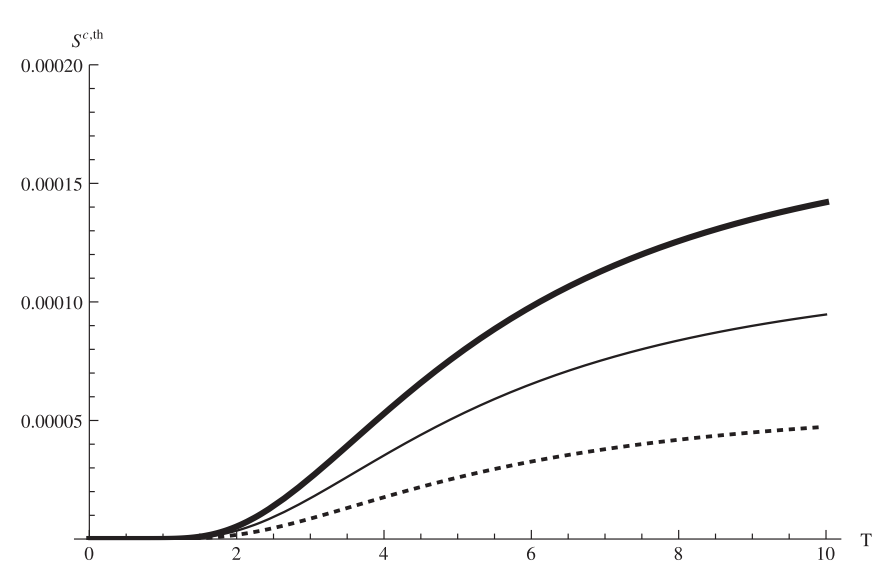

FIG. 2. Plots of the entropy $S^{c \text {,th }}$ of the canonical ensemble vs $T$ with $N=1$ (dotted line), $N=2$ (thin line), and $N=3$ (thick line) for $k_{B}=8.617 \times 10^{-5}$ and $\Delta=0.001$. We see how additivity is clearly preserved and that the limit $T \rightarrow 0$ is equal to zero.

notice that $C_{v}$ increases with $N$ in the limit $T \rightarrow 0$, while for a consistent thermodynamic description it must go to zero. This is a property that is preserved in the usual canonical ensemble. Therefore, its violation cannot be considered a consequence of the quantum nature of the system but an essential property of the SG ensemble that cannot be disregarded.

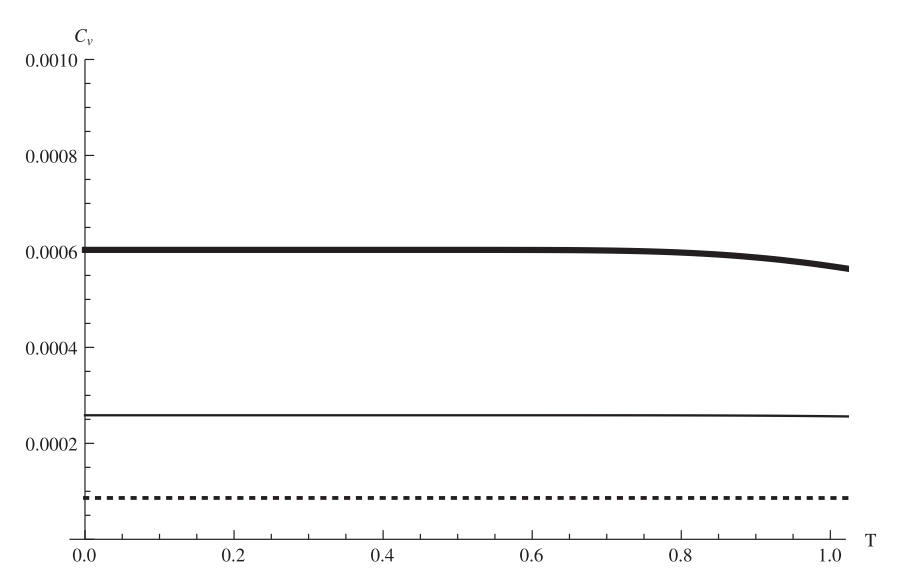

FIG. 3. Plot of the specific heat of the SG ensemble vs $T$ with $N=1$ (dotted line), $N=2$ (thin line), and $N=3$ (thick line) for $k_{B}=8.617 \times 10^{-5}$ and $\Delta=0.001$. We can see that the limit when $T \rightarrow 0$ increases with $N$, while for a consistent thermodynamic description it must go to zero.

\section{Description in terms of density matrices}

\section{Density matrices and von Neumann entropy}

Apart from the description in terms of probability distributions, we can also describe the systems in the previous sections by their corresponding density matrices, using Eq. (2.33). For the simple cases $N=1$ and 2 we obtain

$$
\hat{\rho}_{N=1}^{\mathrm{SG}}=\left(-\frac{\beta \Delta e^{\beta \Delta}-e^{\beta \Delta}+1}{\beta \Delta\left(1-e^{\beta \Delta}\right)}\right) \frac{\left|E_{0}^{1}\right\rangle\left\langle E_{0}^{1}\right|}{\left\langle E_{0}^{1} \mid E_{0}^{1}\right\rangle}-\left(\frac{-\beta \Delta+e^{\beta \Delta}-1}{\beta \Delta\left(1-e^{\beta \Delta}\right)}\right) \frac{\left|E_{\Delta}^{1}\right\rangle\left\langle E_{\Delta}^{1}\right|}{\left\langle E_{\Delta}^{1} \mid E_{\Delta}^{1}\right\rangle}
$$

and

$$
\begin{aligned}
\hat{\rho}_{N=2}^{\mathrm{SG}}= & \left(\frac{2 \beta \Delta+(\beta \Delta-3) \sinh (\beta \Delta)+(\beta \Delta-2) \cosh (\beta \Delta)+2}{2 \beta \Delta[\sinh (\beta \Delta)-\beta \Delta]}\right) \frac{\left|E_{0}^{2}\right\rangle\left\langle E_{0}^{2}\right|}{\left\langle E_{0}^{2} \mid E_{0}^{2}\right\rangle} \\
& +\left(\frac{\beta^{2} \Delta^{2}-2 \cosh (\beta \Delta)+2}{\beta^{2} \Delta^{2}-\beta \Delta \sinh (\beta \Delta)}\right) \frac{\left|E_{\Delta}^{2 a}\right\rangle\left\langle E_{\Delta}^{2 a}\right|}{\left\langle E_{\Delta}^{2 b} \mid E_{\Delta}^{2 a}\right\rangle}+\left(\frac{\beta^{2} \Delta^{2}-2 \cosh (\beta \Delta)+2}{\beta^{2} \Delta^{2}-\beta \Delta \sinh (\beta \Delta)}\right) \frac{\left|E_{\Delta}^{2 b}\right\rangle\left\langle E_{\Delta}^{2 b}\right|}{\left\langle E_{\Delta}^{2 b} \mid E_{\Delta}^{2 b}\right\rangle} \\
& +\left(\frac{2 \beta \Delta-(\beta \Delta+3) \sinh (\beta \Delta)+(\beta \Delta+2) \cosh (\beta \Delta)-2}{2 \beta \Delta[\beta \Delta-\sinh (\beta \Delta)]}\right) \frac{\left|E_{2 \Delta}\right\rangle\left\langle E_{2 \Delta}\right|}{\left\langle E_{2 \Delta} \mid E_{2 \Delta}\right\rangle}
\end{aligned}
$$

where $\left|E_{0}^{1}\right\rangle$ and $\left|E_{\Delta}^{1}\right\rangle$ represent the energy eigenstates for $N=1$ and $\left|E_{0}^{2}\right\rangle,\left|E_{\Delta}^{2 a}\right\rangle,\left|E_{\Delta}^{2 b}\right\rangle$, and $\left|E_{2 \Delta}\right\rangle$ are the eigenstates associated with the $N=2$ case. The vectors $\left|E_{\Delta}^{2 a}\right\rangle$ and $\left|E_{\Delta}^{2 b}\right\rangle$ span the two-dimensional eigenspace with energy equal to $E_{\Delta}$ in the last $N=2$ case.

It is now easy to verify that

$$
\hat{\rho}_{N=2}^{\mathrm{SG}} \neq \hat{\rho}_{N=1}^{\mathrm{SG}} \otimes \hat{\rho}_{N=1}^{\mathrm{SG}} .
$$

It suffices to check that the coefficients of the projectors onto the ground state $\frac{\left|E_{0}\right\rangle\left\langle E_{0}\right|}{\left\langle E_{0} \mid E_{0}\right\rangle}$ do not coincide. This relation translates Eq. (3.9) into the language of density matrices. Indeed, since the Hamiltonian does not introduce a coupling among the different subsystems, the tensor product of equilibrium density states representing single subsystems [such as Eq. (3.14)] should define equilibrium density states of the composite system. This is a property that holds for the usual canonical ensemble in Eq. (2.1), but fails for the SG distribution, as we have just shown.

The SG distribution can also be studied from the point of view of von Neumann's entropy

$$
S^{\mathrm{vN}}=-k_{B} \operatorname{Tr}\left(\hat{\rho}^{\mathrm{SG}} \ln \hat{\rho}^{\mathrm{SG}}\right) .
$$

We know that this quantity is always positive and well defined for any density matrix we evaluate it on. We can also plot (see Fig. 4) the von Neumann entropies for $N=1,2$, and 3 and verify that they are not equally spaced as in the canonical 


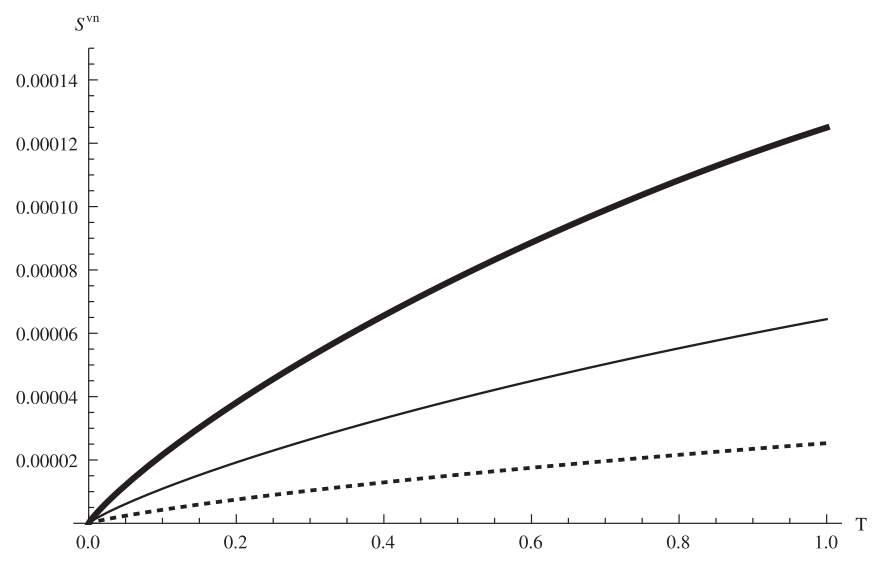

FIG. 4. Plot of von Neumann's entropy of the SG ensemble, for $N=1$ (dotted line), $N=2$ (thin line), and $N=3$ (thick line), vs $T$, with $k_{B}=8.617 \times 10^{-5}$ and $\Delta=0.001$. We can notice that the three levels are not equally spaced. distribution (Fig. 2), where the thermodynamic and the von Neumann entropy functions coincide. Thus, as a consequence of the nonfactorizability of the partition function, the von Neumann entropy is not additive, even if the subsystems are noninteracting. Obviously, by comparing Figs. 4 and 1 we can easily verify that, in the SG case, von Neumann entropy is not the same function as $S^{\text {th }}$, while in the canonical distribution both entropies coincide.

$$
\begin{aligned}
\hat{\rho}_{1}= & \left(\frac{2 \beta \Delta(\beta \Delta-1)+(3-\beta \Delta) \sinh (\beta \Delta)-(\beta \Delta+2) \cosh (\beta \Delta)+2}{2 \beta \Delta[\beta \Delta-\sinh (\beta \Delta)]}\right) \frac{\left|E_{0}\right\rangle\left\langle E_{0}\right|}{\left\langle E_{0} \mid E_{0}\right\rangle} \\
& +\left(\frac{2(\beta \Delta(\beta \Delta+1)+1)-(\beta \Delta+3) \sinh (\beta \Delta)+(\beta \Delta-2) \cosh (\beta \Delta)}{2 \beta \Delta[\beta \Delta-\sinh (\beta \Delta)]}\right) \frac{\left|E_{\Delta}\right\rangle\left\langle E_{\Delta}\right|}{\left\langle E_{\Delta} \mid E_{\Delta}\right\rangle} .
\end{aligned}
$$

Analogously, we can define (and compute)

$$
\hat{\rho}_{2}=\operatorname{Tr}_{1} \hat{\rho}_{N=2}^{\mathrm{SG}}=\hat{\rho}_{1} .
$$

However, we can immediately verify that

$$
\hat{\rho}_{N=2}^{\mathrm{SG}} \neq \hat{\rho}_{1} \otimes \hat{\rho}_{2} \text {. }
$$

This just reflects upon the fact that SG density matrices $\hat{\rho}^{\mathrm{SG}}$ represent entangled states, as we advanced in Sec. IID. Indeed, being defined from partition functions $Z^{\mathrm{SG}}$ that are not factorizable, the description in terms of density matrices $\hat{\rho}^{\mathrm{SG}}$ must also encode this property and it does so in the standard way. We also know that, while separable states have additive von Neumann entropy (see Appendix A for a simple proof), the von Neumann entropy of entangled states is never additive (the difference between the entropy of the composite system with respect to the sum of the entropies of the subsystems is what is called the quantum mutual information in quantum information theory).

\section{CONCLUSION}

In this paper we have proved that the Schrödinger-Gibbs density distribution, defined as the exponential of (minus $\beta$ times) the expected value of the Hamiltonian operator on the corresponding ray, cannot be used to construct a consistent quantum thermodynamics. It has severe problems at the thermodynamical level (the third law of thermodynamics is not satisfied; see, for instance, Fig. 3) and we have devoted some time to show that, in particular, the thermodynamic functions defined by it are nonextensive.

Notice that the definition of the ensemble (2.14) makes perfect sense for finite-dimensional quantum systems, where the integral over the projective space is well defined. The framework we use in Appendix $\mathrm{C}$ to perform the integrals for the partition function and to determine from there the expression of $\hat{\rho}^{\mathrm{SG}}$ [Eq. (2.33)] depends also on the finiteness of the corresponding Hilbert space. A natural question arises: Can we extend our conclusions to general quantum systems, defined on infinite-dimensional spaces? The answer is not simple since the definition itself becomes quite subtle in infinite dimensions because the rigorous mathematical definition of a functional integral over an infinite-dimensional projective space is not an easy task. However, let us assume that the definition of the functional integral is done and let us focus on the generalization of the results. There are two important points to discuss: the procedure we use in Appendix $\mathrm{C}$ and the results we obtain. Obviously the procedure makes sense only if the definition of the integral is done via a limit process over finite-dimensional approximations. In this case we could define an analog of our integrals [Eqs. (C2)-(C9)] for each finite-dimensional approximation without any change. Regarding the results, the limit process would be straightforward as long as the spectrum of the Hamiltonian operator is purely discrete, i.e., if its essential spectrum is empty (see [41,42]). In that case, the dimension of all the eigenspaces is finite and there are no accumulation points in the spectrum. In a situation like this, Eqs. (2.26) and (2.33) make sense even in infinite dimensions.

We conclude thus that the SG ensemble does not make sense as an equilibrium distribution because the thermodynamics associated with it fails to satisfy very basic properties such as additivity or the third law. On the other hand, the use of density distributions is a natural option when studying hybrid quantum-classical models such as Ehrenfest models in nonadiabatic molecular dynamics (see [4,5]). We leave for future work the study of the consequences of the analysis in this paper for the equilibrium and nonequilibrium statistics 
of hybrid quantum-classical systems (see [43] for a recent approach to nonequilibrium and irreversibility), where the nonlinear effects on the dynamics produced by the classical subsystem may alter significantly the results we have presented here.

\section{ACKNOWLEDGMENTS}

We would like to thank Professor Mario Floría for several enlightening discussions that guided us in the right direction. We would also like to thank an anonymous referee for a very careful reading of the manuscript, which has improved it in a very significant manner. This work was supported by Diputación General de Aragón (Spain) Grants No. E24/1, No. E24/2, and No. E24/3; Banco de Santander and Universidad de Zaragoza Grant No. UZ2012-CIE-06; and Ministerio de Economia y Competitividad (Spain) Grants No. FIS201346159-C3-2-P and No. FPA2012-35453.

\section{APPENDIX A: ADDITIVITY OF VON NEUMANN'S ENTROPY}

We will include, for completeness, a classical proof of the additivity of von Neumann's entropy (see, for instance, [44]). Let $\mathcal{S}_{12}=\mathcal{S}_{1}+\mathcal{S}_{2}$ be a composite system with Hilbert space $\mathcal{H}_{12}=\mathcal{H}_{1} \otimes \mathcal{H}_{2}$, of dimension $d_{12}$. We want to prove that the von Neumann entropy is additive on product states $\hat{\rho}_{12}=$ $\hat{\rho}_{1} \otimes \hat{\rho}_{2}$, i.e.,

$$
S\left(\hat{\rho}_{12}\right)=S\left(\hat{\rho}_{1}\right)+S\left(\hat{\rho}_{2}\right),
$$

where $\operatorname{Tr} \hat{\rho}_{12}=1$. Additivity comes from the fact that the spectrum of $\rho_{12}=\rho_{1} \otimes \rho_{2}$ consists of the products of the eigenvalues of $\rho_{1}$ and $\rho_{2}$ :

$$
\begin{aligned}
\hat{\rho}_{1} & =\sum_{i=1}^{d_{1}} \mu_{i}\left|\mu_{i}\right\rangle\left\langle\mu_{i}\left|\Rightarrow \hat{\rho}_{12}=\sum_{k} r_{k}\right| r_{k}\right\rangle\left\langle r_{k}\right| \\
& =\sum_{i=1}^{d_{1}} \sum_{j=1}^{d_{2}} \mu_{i} v_{j}\left|\mu_{i} v_{j}\right\rangle\left\langle\mu_{i} v_{j}\right|, \\
\hat{\rho}_{2} & =\sum_{j=1}^{d_{2}} v_{j}\left|v_{j}\right\rangle\left\langle v_{j}\left|\Rightarrow \hat{\rho}_{12}=\sum_{k} r_{k}\right| r_{k}\right\rangle\left\langle r_{k}\right| \\
& =\sum_{i=1}^{d_{1}} \sum_{j=1}^{d_{2}} \mu_{i} v_{j}\left|\mu_{i} v_{j}\right\rangle\left\langle\mu_{i} v_{j}\right|,
\end{aligned}
$$

where we denote by $\left\{\left|\mu_{i}\right\rangle\right\}_{i=1, \ldots, d_{1}}$ and $\left\{\left|v_{j}\right\rangle\right\}_{j=1, \ldots, d_{2}}$ the eigenvectors of $\hat{\rho}_{1}$ and $\hat{\rho}_{2}$, respectively, and by $\left\{\mu_{i}\right\}_{i=1, \ldots, d_{1}}$ and $\left\{v_{j}\right\}_{j=1, \ldots, d_{2}}$ the corresponding eigenvalues. If we compute von Neumann's entropy in the eigenbasis of $\hat{\rho}_{12}$ we obtain

$$
\begin{aligned}
& S\left(\hat{\rho}_{12}\right)=-k_{B} \sum_{k} r_{k} \ln r_{k}=-k_{B} \sum_{i=1}^{d_{1}} \sum_{j=1}^{d_{2}} \mu_{i} v_{j} \ln \left(\mu_{i} v_{j}\right) \\
& =-k_{B} \sum_{i=1}^{d_{1}} \sum_{j=1}^{d_{2}} \mu_{i} v_{j}\left(\ln \mu_{i}+\ln v_{j}\right) \\
& =-k_{B} \sum_{j=1}^{d_{2}} v_{j} \sum_{i=1}^{d_{1}} \mu_{i} \ln \mu_{i}-k_{B} \sum_{i=1}^{d_{1}} \mu_{i} \sum_{j=1}^{d_{2}} v_{j} \ln v_{j}
\end{aligned}
$$

$$
\begin{aligned}
& =-k_{B} \sum_{i=1}^{d_{1}} \mu_{i} \ln \mu_{i}-k_{B} \sum_{j=1}^{d_{2}} v_{j} \ln v_{j} \\
& =S\left(\hat{\rho}_{1}\right)+S\left(\hat{\rho}_{2}\right),
\end{aligned}
$$

where the fact that $\operatorname{Tr} \hat{\rho}_{1}=\operatorname{Tr} \hat{\rho}_{2}=1$ has been used. As the trace does not depend on the basis, the result is proved.

\section{APPENDIX B: INTEGRALS ON THE PROJECTIVE SPACE}

For the sake of completeness, we prove in this appendix the following result. Consider a function $f$ defined on the sphere $S^{2 n-1} \subset \mathbb{R}^{2 n}$, which is constant along the fibers of the fibration

$$
\tau: S^{2 n-1} \rightarrow \mathbb{C} \mathbb{P}^{n-1},
$$

i.e., which can be obtained as the pullback $\tau^{*}(f)$ of a function $f$ on the projective space or, from the physical point of view, which represents a true physical quantity, as it does not depend on the global phase of the state. Notice that Eq. (B1) corresponds to the restriction of the canonical fibration $\mathbb{C}^{n} \rightarrow \mathbb{C} P^{n-1}$ to the states of norm equal to one. Then we have that

$$
\int_{S^{2 n-1}} d \mu_{S} \tau^{*}(f)=2 \pi \int_{\mathbb{C P}^{n-1}} d \mu_{C} f
$$

where $d \mu_{S}$ and $d \mu_{C}$ represent the corresponding volume forms.

Let us recall that both the sphere and the projective space are nontrivial differentiable manifolds and therefore that integrals on them are obtained by patching together the integrals on the charts of their atlases. Thus, given an open covering $\left\{U_{k}\right\}$ for the manifold $M$, we consider a subordinated partition of unity $\left\{\epsilon_{k}\right\}$, i.e., a collection of functions $\epsilon_{k}: U_{k} \rightarrow \mathbb{R}$ that satisfy that for any point $p \in M$ the sum of the functions corresponding to the open sets to which the point belong is equal to one:

$$
\forall p \in U_{j_{1}} \cap \cdots \cap U_{j_{m}} \Rightarrow \sum_{k=1}^{m} \epsilon_{j_{k}}(p)=1 .
$$

With these, we define the integral on the manifold $M$ as

$$
\int_{M} d \mu(p) F(p)=\sum_{k} \int_{U_{k}} d \mu_{U_{k}}(p) \epsilon_{k}(p) F_{U_{k}}(p),
$$

where $F$ represents a function on $M, d \mu$ represents the corresponding volume element, and $d \mu_{U_{k}}$ and $F_{U_{k}}$ represent the restrictions of the volume element and the function, respectively, to the open set $U_{k}$.

We can now use the bundle structure $\tau: S^{2 n-1} \rightarrow \mathbb{C} \mathbb{P}^{n-1}$ to define a covering for $S^{2 n-1}$ as a product

$$
V_{l m}^{S^{2 n-1}}=U_{l}^{S^{1}} \times U_{m}^{\mathbb{C} \mathbb{P}^{n-1}}
$$

where $\left\{U_{l}^{S^{1}}\right\}$ represent open sets of a covering for $S^{1}$ and $\left\{U_{m}^{\mathbb{C} \mathbb{P}^{n-1}}\right\}$ represent open sets of a covering of the projective space. Such a covering always exists because of the bundle structure. Then consider a partition of unity $\left\{\epsilon_{k}\right\}$ for $\mathbb{C} \mathbb{P}^{n-1}$ associated with the covering defined by the open sets $\left\{U_{k}^{\mathbb{C P}}{ }^{n-1}\right\}$. We can extend this family to define a covering for $S^{2 n-1}$ by considering a partition of the unity $\left\{\varepsilon_{1}, \varepsilon_{2}\right\}$ associated with the 
covering $\left\{W_{1}, W_{2}\right\}$ for the group $U(1) \sim S^{1}$ and defining

$$
\omega_{j k}(p)=\varepsilon_{j}(p) \epsilon_{k}(p) .
$$

It is trivial to verify that $\left\{\omega_{j k}\right\}$ defines a partition of the unity related to the covering $\left\{V_{j k}^{S^{2 n-1}}\right\}$. Next we can define the integral on the sphere as

$$
\int_{S^{2 n-1}} d \mu_{S} \tau^{*}(f)=\sum_{j k} \int_{V_{j k}^{S^{2 n-1}}} d \mu_{V_{j k}} \omega_{j k} \tau^{*}(f)
$$

and, as the integrand is constant on the fibers, we can split the integral in the following way:

$$
\begin{aligned}
\int_{S^{2 n-1}} d \mu_{S} \tau^{*}(f)= & \left(\sum_{j} \int_{j} d \mu_{W_{j}} \varepsilon_{j}\right) \\
& \times \sum_{k} \int_{U^{\mathbb{C P}}{ }_{k}^{n-1}} d \mu_{U_{k}} \epsilon_{k} \tau^{*}(f) .
\end{aligned}
$$

By definition the integral on $U(1)$ is equal to $2 \pi$ and thus we proved that Eq. (B2) holds.

\section{APPENDIX C: THE PARTITION FUNCTION}

Our goal in this appendix is to compute the partition function

$$
Z^{\mathrm{SG}}=\int_{S} d \mu(|\psi\rangle) e^{-\beta\langle\psi \mid \hat{H} \psi\rangle},
$$

where we recall that the relation of the integral on the unit sphere and the integral on the projective space (which is the physically meaningful one) is explained in Appendix B. Consider this distribution written as in Eq. (2.14) and implement the constraint by a complex integral in the form

$$
\begin{aligned}
Z^{\mathrm{SG}}= & \frac{1}{2 \pi} \int_{-\infty}^{\infty} d \lambda \int_{\mathbb{C}^{n}} d \mu(\psi) \\
& \times \exp \left(-\beta \sum_{k} E_{k}\left|\psi_{k}\right|^{2}\right) e^{i \lambda(\langle\psi \mid \psi\rangle-1)} \\
= & \frac{1}{2 \pi} \int_{-\infty}^{\infty} d \lambda e^{-i \lambda} \int_{\mathbb{C}^{n}} d \mu(\psi) \prod_{k=1}^{n} e^{-\left(\beta E_{k}-i \lambda\right)\left|\psi_{k}\right|^{2}},
\end{aligned}
$$

where $d \mu(\psi)=\prod_{k=1}^{n} d \psi_{k} d \bar{\psi}_{k}$ is the canonical volume element in $\mathbb{C}^{n}$. Now the Gaussian integrals factorize and can be computed straightforwardly:

$$
\begin{aligned}
Z^{\mathrm{SG}} & =\frac{1}{2 \pi} \int_{-\infty}^{\infty} d \lambda e^{-i \lambda} \prod_{k=1}^{n} \int_{\mathbb{C}} d \mu\left(\psi_{k}\right) e^{-\left(\beta E_{k}-i \lambda\right)\left|\psi_{k}\right|^{2}} \\
& =\frac{1}{2 \pi} \int_{-\infty}^{\infty} d \lambda e^{-i \lambda} \prod_{k=1}^{n} \frac{2 \pi}{\beta E_{k}-i \lambda}
\end{aligned}
$$

If we also take into account the degeneracies of the eigenvalues we have

$$
Z^{\mathrm{SG}}=(2 \pi)^{n-1} \int_{-\infty}^{\infty} d \lambda e^{-i \lambda} \prod_{k=0}^{p} \frac{1}{\left(\beta E_{k}-i \lambda\right)^{d_{k}}},
$$

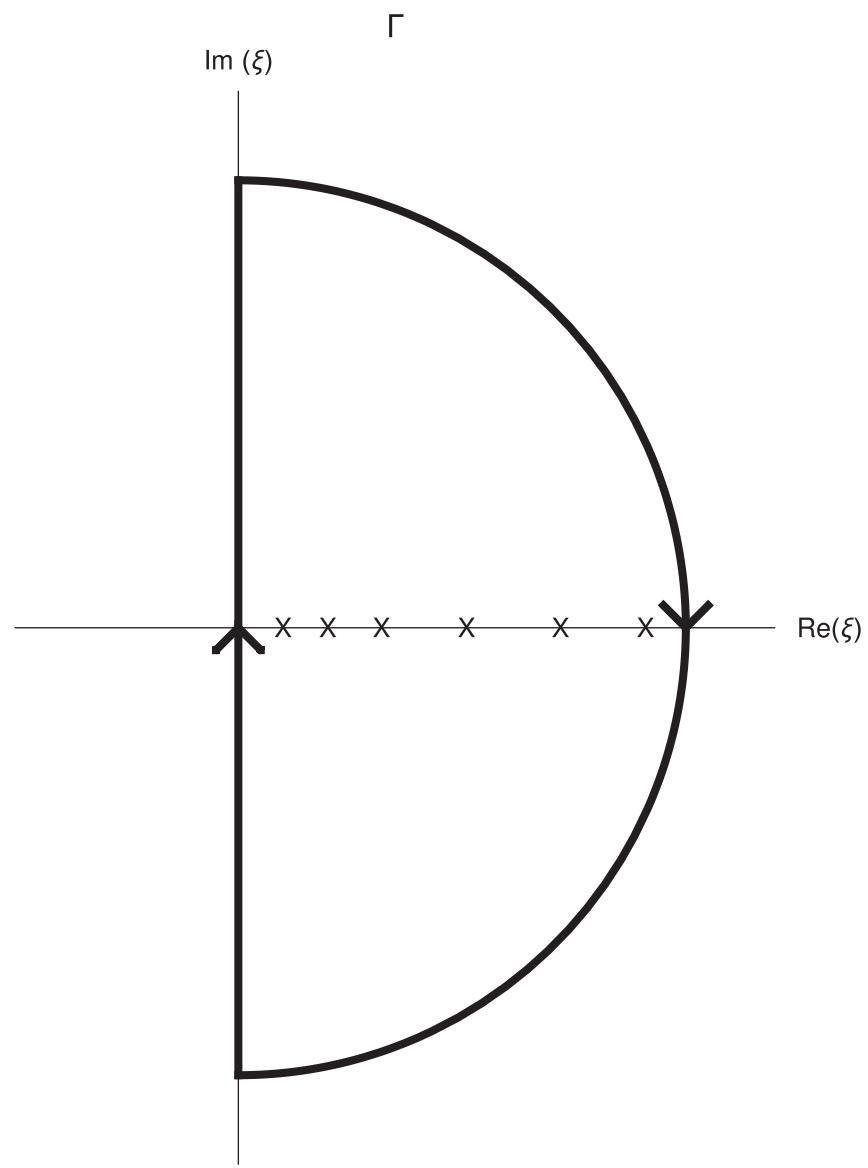

FIG. 5. Representation of the integration region $\Gamma$ in the complex plane. The poles are represented on the real axis.

where $d_{k}$ represents the degeneracy of eigenvalue $E_{k}$ and the product runs only over different eigenvalues (we assume that there are $p+1$ of them).

The last integral (over $\lambda$ ) must be evaluated on the complex plane, where the integration runs over the real axis. To this end, let us first make a change of coordinates

$$
\xi=i \lambda
$$

that produces

$$
Z^{\mathrm{SG}}=i(2 \pi)^{n-1} \int_{\gamma} d \xi e^{-\xi} \prod_{k=0}^{p} \frac{1}{\left(\beta E_{k}-\xi\right)^{d_{k}}},
$$

where now $\gamma$ is the imaginary axis.

Next we define a closed integration region $\Gamma$ on the left half plane containing the imaginary axis and a semicircle of infinite radius as depicted in Fig. 5. From the Cauchy theorem, we know that the integral is equal to the sum of the corresponding residues at the poles of the integrand, which in this case lie on

$$
\xi_{k}=\beta E_{k},
$$

their order corresponding to the degeneracy of the corresponding eigenvalue. We have thus that

$$
Z^{\mathrm{SG}}=i(2 \pi)^{n-1} 2 \pi i \sum_{j=0}^{p} \operatorname{Res}\left(\frac{e^{-\xi}}{\prod_{k=1}^{p}\left(\beta E_{k}-\xi\right)^{d_{k}}}, \xi=\beta E_{j}\right) .
$$


Then we can write

$$
Z^{\mathrm{SG}}=\sum_{k=0}^{p} e^{-\beta E_{k}} F_{E_{k}}
$$

where

$$
F_{E_{k}}=-\left.(2 \pi)^{n} e^{\beta E_{k}} \frac{1}{\left(d_{k}-1\right) !}\left[\frac{\partial^{d_{k}-1}}{\partial \xi^{d_{k}-1}}\left(e^{-\xi} \prod_{\substack{s=0 \\ s \neq k}}^{p} \frac{1}{\left(\beta E_{s}-\xi\right)^{d_{s}}}\right)\right]\right|_{\xi=\beta E_{k}}
$$

By computing the derivative we obtain

$$
F_{E_{k}}=-\frac{(2 \pi)^{n}}{\left(d_{k}-1\right) !} \sum_{\substack{j_{0}, \ldots, j_{p}=0 \\
j_{0}+j_{1}+\cdots+j_{p}=d_{k}-1}}^{d_{k}-1}\left(\begin{array}{c}
d_{k}-1 \\
j_{0}, \ldots, j_{p}
\end{array}\right) \prod_{\substack{s=0 \\
s \neq k}}^{p} \frac{(-1)^{j_{0}}\left[\left(d_{s}+j_{s}-1\right) ! /\left(d_{s}-1\right) !\right]}{\left(\beta E_{s}-\beta E_{k}\right)^{d_{s}+j_{s}}},
$$

where $\left(\begin{array}{c}d_{k}-1 \\ j_{0}, \ldots, j_{s}\end{array}\right)$ represents the multinomial coefficient

$$
\left(\begin{array}{c}
d_{k}-1 \\
j_{0}, \ldots, j_{s}
\end{array}\right)=\frac{\left(d_{k}-1\right) !}{j_{0} ! \cdots j_{s} !} .
$$

This concludes the proof.

[1] E. Schrödinger, Statistical Thermodynamics (Courier Dover, Mineola, 1967).

[2] A. I. Khinchin, Mathematical Foundations of Quantum Statistics (Graylock, Toronto, 1960), Chap. III, Sec. 2.

[3] L. D. Landau and E. M. Lifshitz, Statistical Physics, translated from Russian by E. Peierls and R. F. Peierls, Course of Theoretical Physics Vol. 5 (Pergamon, London, 1959).

[4] J. L. Alonso, A. Castro, J. Clemente-Gallardo, J. C. Cuchí, P. Echenique, and F. Falceto, Statistics and Nosé formalism for Ehrenfest dynamics, J. Phys. A: Math. Theor. 44, 395004 (2011).

[5] J. L. Alonso, J. Clemente-Gallardo, J. C. Cuchi, P. Echenique, and F. Falceto, Ehrenfest dynamics is purity non-preserving: A necessary ingredient for decoherence, J. Chem. Phys. 137, 054106 (2012).

[6] J. Von Neumann, Mathematical Principles of Quantum Mechanics (Princeton University Press, Princeton, 1955).

[7] D. C. Brody and L. P. Hughston, The quantum canonical ensemble, J. Math. Phys. 39, 6502 (1998).

[8] C. M. Bender, D. C. Brody, and D. W. Hook, Solvable model of quantum microcanonical states, J. Phys. A: Math. Gen. 38, L607 (2005).

[9] D. C Brody, D. W Hook, and L. P. Hughston, On quantum microcanonical equilibrium, J. Phys.: Conf. Ser. 67, 012025 (2007).

[10] G. Jona-Lasinio, Invariant measures under Schrödinger evolution and quantum statistical mechanics, Can. Math. Soc. Conf. Proc. 20, 239 (2000).

[11] G. Jona-Lasinio and C. Presilla, On the statistics of quantum expectations for systems in thermal equilibrium, AIP Conf. Proc. 844, 200 (2006).

[12] J. Naudts and E. Van der Straeten, A generalized quantum microcanonical ensemble, J. Stat. Mech. (2006) P06015.
[13] S. Goldstein, J. L. Lebowitz, R. Tumulka, and N. Zanghì, On the distribution of the Wave function for systems in thermal equilibrium, J. Stat. Phys. 125, 1193 (2006).

[14] B. Fresch and G. J. Moro, Emergence of equilibrium thermodynamic properties in quantum pure states. I. Theory, J. Chem. Phys. 133, 034509 (2010).

[15] B. Fresch and G. J. Moro, Typicality in ensembles of quantum states: Monte Carlo sampling versus analytical approximations, J. Phys. Chem. A 113, 14502 (2009).

[16] B. V. Fine, Typical state of an isolated quantum system with fixed energy and unrestricted participation of eigenstates, Phys. Rev. E 80, 051130 (2009).

[17] B. V. Fine and F. Hantschel, An alternative to the conventional micro-canonical ensemble, Phys. Scr. T 151, 014078 (2012).

[18] K. Ji and B. V. Fine, Nonthermal statistics in isolated quantum spin clusters after a series of perturbations, Phys. Rev. Lett. 107, 050401 (2011).

[19] M. P. Müller, D. Gross, and J. Eisert, Concentration of measure for quantum states with a fixed expectation value, Commun. Math. Phys. 303, 785 (2011).

[20] M. Campisi, Quantum fluctuation relations for ensembles of wave functions, New J. Phys. 15, 115008 (2013).

[21] L. Campos Venuti and P. Zanardi, Probability density of quantum expectation values, Phys. Lett. A 377, 1854 (2013).

[22] E. Gutkin and K. Życzkowski, Joint numerical ranges, quantum maps, and joint numerical shadows, Linear Algebra Appl. 438, 2394 (2013).

[23] K. Huang, Statistical Mechanics, 2nd ed. (Wiley, New York, 1987).

[24] L. E. Reichl, A Modern Course in Statistical Physics (Wiley, New York, 1998).

[25] H. B. Callen, Thermodynamics and an Introduction to Thermostatistics (Wiley, New York, 2006). 
[26] There is an ambiguity in the definition of the volume element on the sphere by using the constraint, but it represents just a global multiplicative constant, which is irrelevant to defining the corresponding statistical system.

[27] R. B. Griffiths, Consistent Quantum Theory (Cambridge University Press, Cambridge, 2003).

[28] P. C. Hohenberg, Colloquium: An introduction to consistent quantum theory, Rev. Mod. Phys. 82, 2835 (2010).

[29] E. T. Jaynes, Information theory and statistical mechanics II, Phys. Rev. 108, 171 (1957).

[30] C. A. Fuchs, Distinguishability and accessible information in quantum theory, Ph.D. thesis, University of New Mexico, 1995, arXiv:quant-ph/9601020v1.

[31] C. Cafaro and P. van Loock, Towards an entropic analysis of quantum error correction with imperfections, AIP Conf. Proc. 1553, 275 (2013).

[32] M. A. Nielsen and I. L. Chuang, Quantum Computation and Quantum Information (Cambridge University Press, Cambridge, 2010).

[33] A. Peres, Quantum Theory: Concepts and Methods (Kluwer, Dordrecht, 1995).

[34] K. Maruyama, N. Franco, and V. Vedral, Colloquium: The physics of Maxwell's demon and information, Rev. Mod. Phys. 81, 1 (2009).
[35] S. W. Kim, T. Sagawa, S. De Liberato, and M. Ueda, Quantum Szilard Engine, Phys. Rev. Lett. 106, 070401 (2011).

[36] M. Plesch, O. Dahlsten, J. Goold, and V. Vedral, Comment on "Quantum Szilard Engine", Phys. Rev. Lett. 111, 188901 (2013).

[37] S. W. Kim, K.-H. Kim, T. Sagawa, S. De Liberato, and M. Ueda, Kim et al., Reply, Phys. Rev. Lett. 111, 188902 (2013).

[38] D. C. Brody, L. P. Hughston, and M. F. Parry, Effects of quantum entanglement in phase transitions, Phys. Lett. A 374, 2424 (2010).

[39] For simplicity we will consider the case of nondegenerate ground states.

[40] See Supplemental Material at http://link.aps.org/supplemental/ 10.1103/PhysRevE.91.022137 for the Mathematica notebook for the computation of the functions at any order (results are too long to detail them here for $N>2$ ).

[41] S. Sternberg, Theory of Functions of a Real Variable (University Press of Florida, Gainesville, 2005).

[42] E. B. Davies, Linear Operators and Their Spectra (Cambridge University Press, New York, 2007).

[43] G. Gallavotti, Nonequilibrium and irreversibility, Theor. Math. Phys. (2014), doi:10.1007/978-3-319-06758-2.

[44] A. Wehrl, General properties of entropy, Rev. Mod. Phys. 50, 221 (1978). 